\title{
Multi-Token Based MAC-Cum-Routing Protocol for WSN: A Distributed Approach
}

\author{
Subhasis Dash, Kumar Saras, Manas Ranjan Lenka, and Amulya Ratna Swain
}

\begin{abstract}
A wireless sensor network is a collection of batterypowered sensor nodes distributed in a geographical area. There are many applications, where networks are left unattended for a long period of time. These networks suffer from problems like high energy consumption, high latency time, and end-to-end low packet delivery ratio. To design a protocol that finds a trade-off between these problems is a challenging task. In order to mitigate the above issues, different existing Media Access Control (MAC) protocols such as S-MAC, RMAC, HEMAC, Congestion-less Single Token, AS2-MAC, and Multi Token based MAC protocols have been proposed which ensure better packet delivery but fail to ensure energy efficiency due to high end-to-end latency. The problem of high end-to-end latency is resolved with the existing routing protocols such as Fault Tolerant Multilevel Routing protocol(FMS), and Enhanced Tree Routing protocol(ETR). So, it is clear that MAC and routing protocols both together can give better results related to data transmission in WSN. In order to achieve the same, in this paper, we propose a MAC-cumRouting protocol collision free data Transmission, which improves throughput with respect to end to end packet delivery. The proposed protocol exchanges various control messages among the sensor nodes in a distributed manner to achieve better data packet delivery and ultimately uses tokens to ensure collision free data transmission. Simulation studies of the proposed approach have been carried out and its performance has been compared with the Multi Token based MAC protocol, AS-MAC protocol, and ETR routing protocol. The experimental results based on simulation confirms that the proposed approach has a higher data packet delivery ratio.
\end{abstract}

Index Terms-Wireless Sensor Network, Hierarchical Network Structure, Energy Efficiency, Routing, MAC.

\section{INTRODUCTION}

Wireless Sensor Network consists of a large number of sensor nodes equipped with various sensing devices, which are spatially spread over a hostile environment. These sensor nodes monitor physical or environmental conditions such as temperature, pressure, sound, etc., and communicate among themselves using wireless channels only. These sensor nodes are operated at low power with limited processing speed and storage capacity. Sensors nodes are battery powered and can run only for 100-120 hours with AAA batteries when these nodes work in active mode. Hence, the major concern while

Manuscript received January 2, 2019; revised July 29, 2019. Date of publication September 27, 2019. Date of current version September 27, 2019.

S. Dash, M. R. Lenka and A. R. Swain are with the School of Computer Engineering, KIIT Deemed to be University, Bhubaneswar, India, e-mails: \{subhasisbbsr, saraskiit21, manasy2k3, swainamulya\}@gmail.com

K. Saras is with SAP Labs India, Bangalore, India e-mail saraskiit21@gmail.com.

Digital Object Identifier (DOI): 10.24138/jcomss.v15i3.709 designing a protocol for WSN is to reduce energy consumption as in many applications sensor nodes are deployed in inaccessible and unattended environments.

Many event driven applications of WSN require critical data detected by the sensor nodes which need to be transmitted to the sink node in a collision free manner. However, the collision free data transmission in WSN always consumes more energy. In the view of above, optimization of energy consumption is always considered as one of the basic issues in the design of any MAC or routing protocols related to WSN. The optimization of energy consumption in WSN can be taken care of during the selection of a data transmission path by involving a minimum number of nodes in-between source and sink. Each sensor node present in the network must equally participate for data transmission in order to dissipate the equal amount of energy from each node which increases the network lifetime [1]. However, at the same time, the data routing within the sensor network with minimum latency time is also another challenging issue in the design of MAC protocols [2]. In order to prolong the network lifetime of WSN, there are various sources of energy wastage which need to be considered while designing a MAC or Routing protocol. Various sources of energy wastage are as follows:-

1) Overhearing: It is a condition when a packet destined for a particular node is received by other neighboring nodes and these nodes waste energy in receiving this radio.

2) Collision: When two nodes try to access the shared radio channel at the same point of time then a collision occurs. It is a very common phenomenon in a shared channel, but it increases energy consumption and packet latency.

3) Idle Listening: When an inactive sensor leaves its RX turned on to listen to the carrier signal, it leads to energy wastage which is the same in amount as the energy dissipated during a normal reception.

There are a number of existing MAC and routing protocols which prolong the lifetime of sensor nodes and also maintain data transmission with high throughput. These protocols include S-MAC [3]-[5], RMAC [6], HEMAC [7], token based MAC [8]-[16] and Routing protocols [17]-[29], Energy Aware Routing Protocol with Sleep Scheduling [30]-[40] which are basically designed on top of a hierarchical structure.

The Self Organizing Message passing MAC protocol [12] ensures less collision of data packets by introducing request, reply, and token (at sink node) as control packets for collision free data transmission. However, the protocol suffers from loss of network connectivity, even if the alternate parent node has 
not yet run out of energy due to the use of the same network path for a large amount of data transmissions. Congestionless Single Token MAC protocol [9] tries to overcome the above drawback by selecting alternative network paths from time to time during data transmission. Nevertheless, it could not manage the network hole in case both the parent nodes runs out of energy. Fault Tolerant Multilevel Routing protocol (FMS) [10] takes care of the above issue through discovery of alternate parents and continues the data transmission in an uninterrupted manner that ultimately leads to a better data packet delivery ratio. All these above protocols still suffer from high end-to-end latency due to the use of a single token which passes from the sink to the leaf node to make the data transmission feasible.

Considering these above issues, a Multi-Token based MAC protocol [11] was designed that uses multiple tokens available at immediate parent nodes instead of the sink node in a sensor network to achieve parallel data transmission with reduced latency. In Multi Token based MAC protocol [11], the network structure changes in a periodic manner to consume a balanced amount of energy from each node during data transmission. At the same time, the protocol suffers from high energy consumption during network reconstruction. The problem of high energy consumption during network reconstruction was reduced in Distributed Hierarchical Structure Routing protocol [41]. However, in the protocol [11] as mentioned above, the loss of data and control packets due to improper token management affects the data throughput.

In order to improve the performance related to data throughput, a MAC-cum-routing protocol is proposed here that uses a robust token distribution technique for collision free data transmission along with the distributed approach mentioned in [41] to support better energy consumption.

The rest of the paper is organized as follows. Section II presents a survey of related works along with the motivation of our proposed work. The proposed protocol is described in section III. Performance evaluation of the proposed protocol and its comparison with existing protocols is given in section IV. Section V concludes the paper.

\section{RELATED WORK}

The major challenges faced by Wireless Sensor Networks (WSNs) during the design of MAC and Routing protocols are limited energy resource, end-to-end transmission latency, throughput, etc. In order to mitigate the difficulties to overcome above challenges for WSNs, the design approach to have a new protocol must look into some of the usual constraints such as high energy consumption, data packet collision, and lack of reliability in communication. Loss of token and other control packets during transmission due to collision increases energy consumption and data transmission latency. Energy consumption during reliable data communication varies with network structure. There are basically two types of network structures, viz. flat structure and hierarchical structure. The Flat structure uses routing techniques like flooding, directed diffusion, etc. The hierarchical structure gets an edge over the flat structure in terms of scalability and efficiency. There are many existing Routing and MAC protocols which are based on the hierarchical structure and deals with such problems.

Ye et al. proposed a contention based protocol known as energy efficient Sensor-MAC (S-MAC) [3], [4] protocol. It integrates both low duty-cycle and multi-hop operations. It also constructs an organized network topology with the low dutycycle operation and tries to send a maximum number of data packets from different sensor nodes to the sink node in a single duty cycle. Due to a large number of data packets being sent to the sink node at the same time, it leads to heavy traffic at the sink node which in turn leads heavy data packet collision. In order to reduce energy consumption, it also integrates a periodic listen and sleep mechanism. At the same time, the protocol suffers from high end-to-end latency in data packet delivery due to fixed listen and sleep period.

$\mathrm{Du}$ et al. proposed a Routing enhanced MAC protocol (RMAC) [6] that uses a hierarchical structure for data transmission and uses different routing information to reduce energy consumption. In this protocol, before data transmission gets started, a control frame called PION moves across different level of hops and fixes the path for the upcoming data packet. Initially, all the intermediate sensor nodes are in sleep mode but wake up intelligently when an event is detected. Only the nodes that relayed the PION can wake up during the sleep period to relay the data packet. These nodes do not go back to sleep until the next sleep period starts again.

Cho and Bahk proposed a Hop Extended MAC protocol (HEMAC) [7] that tries to utilize a single duty cycle during data transmission to cover maximum hops. It uses EXP (Explorer) control packet to store information about intermediate nodes belonging to the network path through which data packet is transmitted over maximum hops in a single duty cycle. The same EXP control packet is also used to set up nodes to forward upcoming data packets during the data period of the duty cycle. The active radio state of each node does not change at the beginning of sleep period as per the information stored in the EXP frame. This ensures the relay of the EXP frame beyond the start of the sleep period. The protocol ensures reduced energy consumption by adaptive sleeping in the data period.

Swain et al. proposed an Energy Aware Routing Protocol [42] with Sleep Scheduling in order to reduce energy consumption in WSNs. The protocol identifies intermediate and leaf nodes during the construction of the hierarchical structure. As per the design of this protocol, the leaf nodes are put to sleep while the intermediate nodes remain awake after the construction of a hierarchical structure. The hierarchical structure is periodically reconstructed considering the remaining energy of each node to ensure balanced energy consumption over the sensor network. The protocol also ensures fault tolerance in case of node failures. The protocol suffers from heavy data packet collision which increases energy consumption by the WSN.

In the recent past, SandhyaSree et al. proposed a Crosslayer Token Passing mechanism [14] that combines localization technique of routing and token passing approach for transmission of data packets to the sink node. It first divides the sensor network into levels by broadcasting messages from the 
sink node at different power levels, so that such broadcasting messages can reach one or more sensor nodes in the network. It then divides the network into equiangular sectors (groups) in the clockwise or anticlockwise direction. A token is assigned to a node in each group and that node can hold the token for a fixed amount of time after which it passes the token to a neighboring node. A node can only transmit data packets when it holds the token. A sensor node forwards data only if it comes from a higher level and from an adjacent sector to ensure transmission only towards the base station and not away from it. This protocol suffers from the problem of high end-to-end latency as a single token is assigned to each group for data transmission.

Self Organizing Message passing MAC protocol [12] for WSNs proposed by Ray et al. is a protocol designed for multi-hop WSN which ensures end-to-end data delivery by using a single token based approach. It uses different control packets like request and reply packet for collision free data transmission. The token is only available at the sink node. The sink node also maintains a request queue to keep track of the request messages according to the time-stamp of the request from the source node and provides the token in first come first serve basis. A source node can transmit data only after receiving the token from the sink node. It ensures collision free data transmission but increases the packet delivery latency, which consequently increases the energy consumption. After a node transmits data, it has to return back the token with the last data packet to the sink node so that the token can be allocated to another node present in the request queue. As per the protocol, the same set of intermediate nodes is used as a transmission path for a long period of time which may lead to network hole.

Ray et al. proposed a Congestion-less Single Token based MAC protocol [9] that uses a single token for data transmission and it ensures minimum data collision. In this approach, the transmission path is always altered after a fixed amount of time to ensure balanced power consumption over intermediate nodes. The protocol suffers from the problem of high end-toend latency due to the use of the single token approach. It also suffers from loss of network connectivity in case of node failure or when a node runs out of energy.

Ajay et al. proposed a Fault Tolerant Multilevel Routing protocol (FMS) [10] that uses a token based approach which ensures data accuracy and the minimum number of collision during data transmission. In addition, the protocol is fault tolerant and dynamic enough to handle failures when the node dies out. Each intermediate dying node sends an EnergyLow packet to its respective child nodes for selection of new parent node in order to achieve network connectivity. The time taken to find a new neighbor, when a node failure occurs, affects the delivery of data packets. At the same time, the protocol suffers from the problem of end-to-end latency as the single token is used for the whole network.

Wanzhi et al. proposed an Enhanced Tree Routing protocol (ETR) [43] that uses an alternative one-hop neighbour shortest path in addition to parent \& child link. Nevertheless, at the same time, the protocol has another challenge to find out the alternative shortest path from the information contained in the neighbor table and their address structure. The protocol uses the default parent-child link to forward the data packet to its next hop, but ETR algorithm enables a neighbor node to find an alternative shortest path link to forward the data packet to the sink node. Thus the protocol gives better end-to-end data delivery with minimum energy, however finding an alternate path is too costly in terms of storage space and computing time.

Dash et al. proposed a Multi Token based MAC protocol [11] that prolongs network lifetime and also ensures network connectivity. The protocol periodically reconstructs the hierarchical structure to achieve better utilization of energy resources. It also integrates random sleep scheduling to ensure balanced energy consumption. At the same time, it ensures fault tolerance for network connectivity and congestion less data transmission even if a node dies out.

Luca et al. proposed an AS2-MAC protocol [44] with a smart scheduler which tries to wake up a node to send and receive the data packet in any duty cycle period. At the same time, the scheduler policy enables the radio of a node to transmit and receive data packets only when it is required to communicate with their neighbors, which in turn reduces idle listing and overhearing. Periodically, the MAC protocol itself alert for incoming data transmission over each channel(s) with low duty cycle. This ensures hop-to-hop data delivery with minimum energy consumption.

In recent past Dash et al. proposed a Distributed Hierarchical Structure Routing protocol [41] to design the hierarchical network structure for a sensor network in a distributed manner. This protocol considers that the functionalities of each sensor node can be used independently and parallel while constructing the hierarchical network structure. It reduces both time and energy during the reconstruction of the hierarchical structure. The protocol also ensures that less number of intermediate nodes gets selected during the reconstruction

From the above discussion, it is clear that network structure construction, energy consumption, network connectivity and collision free data transmission are major issues in WSNs. All the above MAC and routing protocols suffer from the problem of collision free data transmission leading to low delivery ratio. Our proposed protocol ensures collision free data transmission and the high delivery ratio by handling different categories of packet loss such as Request loss, Token loss, Reply loss and Acknowledgment loss. Each intermediate node (parent node) in the network is assigned with a token which can be allocated to its child nodes to achieve collision-free data transmission and reduce end-to-end latency. The accumulated data is sent together using a minimum number of control packets which reduces congestion and energy consumption during data transmission. Also, the hierarchical structure is reconstructed periodically to ensure balanced energy consumption over the sensor network which helps in avoiding loss of network connectivity.

\section{PROPOSED APPROACH}

In this paper, we propose a collision free data transmission approach over a distributed network structure using different 
control packets for improving end-to-end data delivery ratio. The distributed network structure is found to be suitable for developing MAC or Routing protocols. In addition to this, the control packets significantly regulate the data packets with ensuring high delivery ratio during data transmission. In order to achieve effective energy consumption for WSN, the proposed approach considers the sensor nodes of the WSN to be static. The sensor nodes are densely and randomly deployed. The information gathered by them is required to be transmitted to the sink node. Each sensor node is assumed to be assigned a unique ID and the communication between them are symmetric and bidirectional. Furthermore, it is assumed that all the sensor nodes have limited resources, while the sink node is more powerful to perform any task and communicate with all the sensor nodes directly or indirectly in the network. It is also assumed that each node maintains a buffer to store data packets and also maintains a queue to store request messages coming from its lower level nodes or child nodes. The major objectives of the proposed protocol are as follows:

1) The data transmission should be more reliable.

2) Increased packet delivery ratio with less energy consumption as each sensor node is powered with limited energy.

In this proposed approach, first, we construct a hierarchical tree structure using the distributed technique given in [41]. According to the distributed technique used for the construction of a hierarchical structure, each node can be used independently and parallel during tree construction. Once the tree construction gets over, a child discovery packet is transmitted by each node to its parent nodes. This packet is used by each node to identify itself as an intermediate or leaf node. The leaf nodes, which are expected to be very high in number, are put to sleep and the intermediate nodes remain awake. Each intermediate node is allocated with a token that works as permission to transmit the data packet. If a node detects any events, it requests for a token to both of its parent nodes. After the exchange of various control messages, the node accepts the token from only one of its parent node and transmits the data packet to the parent node from whom it has accepted the token. The parent node also follows the same process to transmit the data packet to its parents and finally, the data gets reached sink node. The tree is constructed from time to time in such a way that the nodes that have higher remaining energy are selected as the intermediate nodes. So that the data/control packets will be transmitted through the intermediate nodes which have more energy. The tree is periodically reconstructed in order to ensure that the consumption of energy by each node remains balanced. As outlined above, the proposed routing protocol consists of three phases which are as follows:

1) Tree Construction Phase

2) Token Management Phase

3) Data Transmission Phase

\section{A. Tree Construction Phase}

In this phase, all the sensor nodes in WSN are segregated into two parts, i.e., intermediate nodes and leaf nodes. The

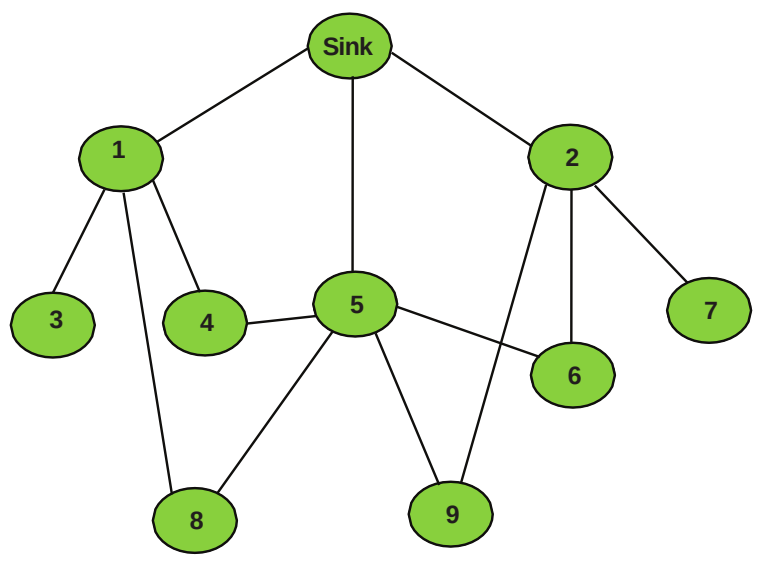

Fig. 1. The hierarchical tree structure

number of intermediate nodes selected during the tree construction should be as minimum as possible. The tree construction should be done periodically so that the intermediate nodes are changed from time to time, which prolong the network lifetime. Also, the time and energy consumed during the construction of the tree should be as minimum as possible. The tree construction is done as per the approach is given in Distributed Hierarchical Structure Routing protocol [41] that satisfies the required features for the proposed protocol. The initial phase of tree construction, i.e., the level discovery phase is done in a sequential manner to identify the level of each node present in WSN. Whereas other phases of tree construction such as energy discovery and parent discovery phase are completely distributed in nature. This distributed approach reduces both the time and energy required during tree construction. During the energy discovery phase, each node discovers remaining energy information from its neighboring nodes and stores information about any two of its neighboring nodes from the same level and two from the immediate lower level having maximum remaining energy. During the parent discovery phase, a node first decides to be active or inactive based on the energy information of the two neighboring nodes of the same level stored during the energy discovery phase. If a node has higher remaining energy as compared to its neighboring nodes, then the node remains in the active state otherwise, it moves to an inactive (sleep) state. The node which goes to sleep state keeps the information of both the parent nodes intact.

Figure 1 shows the hierarchical tree structure which is formed during the tree construction phase. From this figure, it can be seen that each node, i.e., node 1 to node 9 , has either one parent node or two parent nodes which are useful for transmitting the data packets towards the sink node.

\section{B. Token Management Phase}

In this proposed protocol, the token management phase is executed immediately after the tree construction phase. A token is the most important control packet required during data transmission. A sensor node can transmit data only if it has acquired a token from its parent node. This phase decides the nodes which are eligible to possess token and how the 


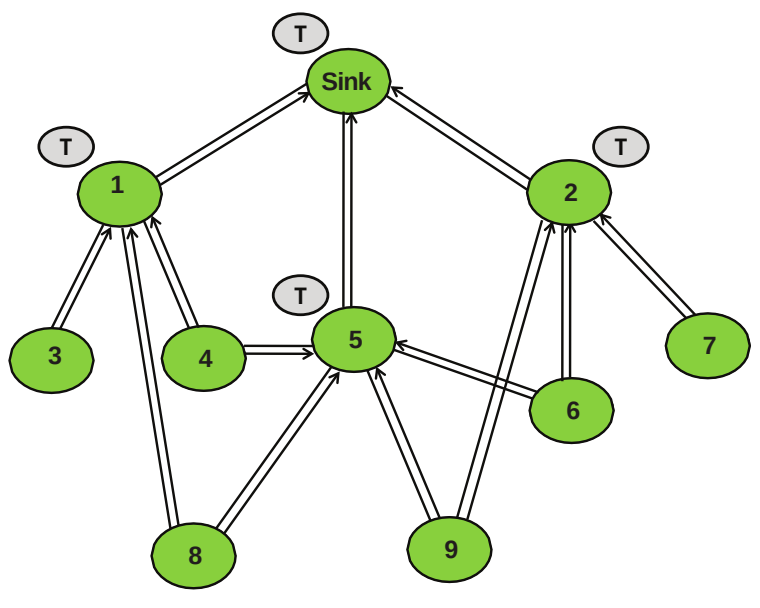

Fig. 2. Transmission of request and token message in the network

token gets allocated to a requesting node. It also describes the procedure by which a node can request for a token to its parent nodes, acquire it, transmit the data, and return back the token to its parent node.

After the tree construction phase, the intermediate nodes have no idea of whether they are parents of any other nodes or not. According to the proposed algorithm, only the intermediate nodes will keep the token and the token request will start only from the leaf nodes. Hence, there is a need for segregating the nodes, i.e., either an intermediate or leaf node. In order to distinguish the nodes, at the beginning of the token management phase, each node sends a child discovery packet to each of its parent nodes that are selected during the tree construction phase. This packet will help to identify whether a node is intermediate or leaf node. Each intermediate node is assigned a token which it can allocate to a child node for the data transmission.

In order to ensure collision free data transmission, a token can be allocated to only one child node at a time. This reduces the collision of data packets during data communication. In order to transmit data, each source node acquires token from its parent node and this process continues till all the data packets reach the sink node. Data packets are accumulated at each of their intermediate nodes and these accumulated data packets are then transmitted, which reduces the collision in the network. If multiple token request packets from different child node arrive at an intermediate node, then the token is granted to only one child node based on the arrival time of the request packet in first come first serve basis. The requesting node first accepts the granted token from any one of its parents and then sends a reply message to the parents. At last, the parent, whose token was accepted, sends back an acknowledgment message to the requesting node before the data packet gets transmitted. Each token is granted for a fixed amount of time, after that the child node has to return back the token to the intermediate parent node.

Figure 2 shows the transmission of request packets from child nodes to their parent nodes. When the leaf nodes viz. $3,4,6,7,8,9$, in the figure detect an event, they send a request packet to their parent nodes. The parent nodes (like 1 ,

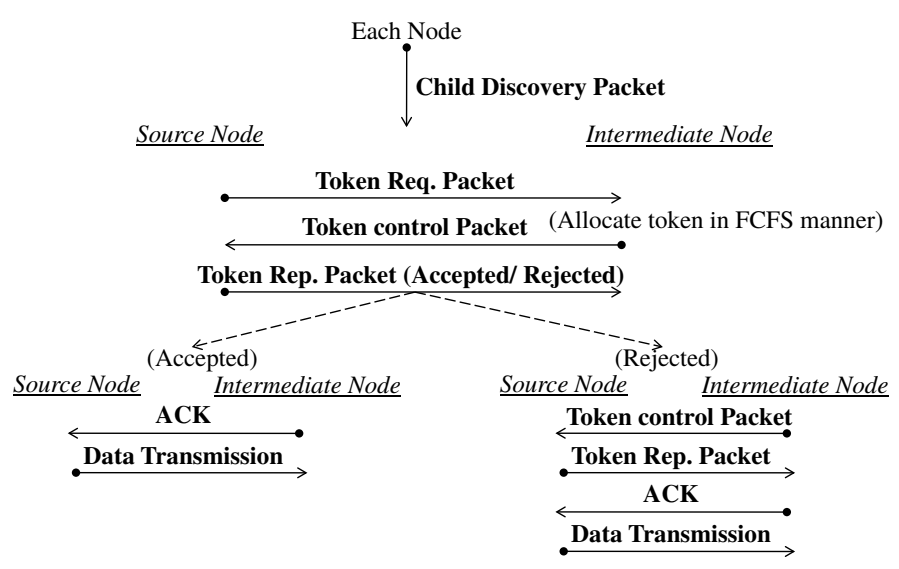

Fig. 3. Control flow for token management

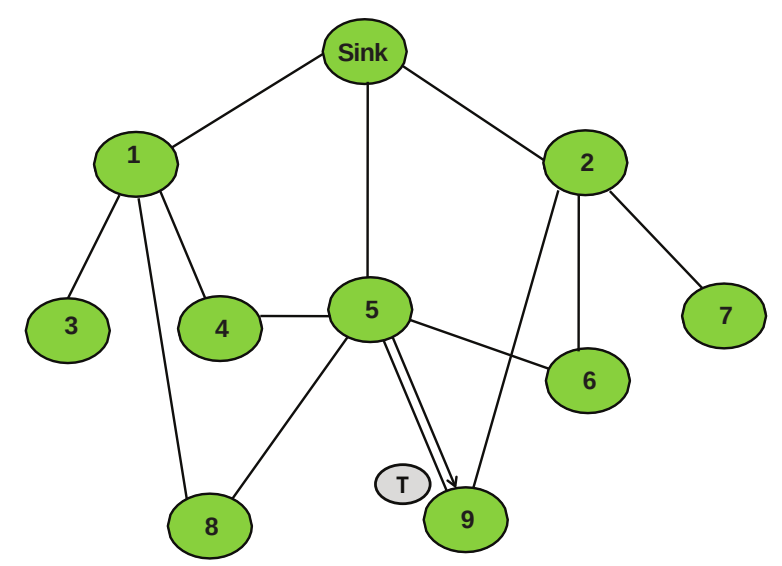

Fig. 4. Child node receives a token message from its parent node

$5,2)$ in turn send request packets to their parent nodes. This process continues until the request packet reaches the sink node. Each parent (or intermediate) node is allocated with a token which it assigns to the requesting child node for a limited amount of time to transmit their data packets. This request packet management is given in Algorithm 1.

According to the proposed protocol, four control messages are required for data transmission to take place between two nodes, namely, request packet, the token control packet, Reply packet, and Acknowledgment packet. Request messages are sent from a child node $\left(N_{j}\right)$ to its intermediate parent nodes $\left(P_{j 1}\right.$, and $\left.P_{j 2}\right)$. When a parent node, either $P_{j 1}$ or $P_{j 2}$ or both, receives request packets from different child nodes, it allocates its token based on the arrival time-stamp of requesting node by releasing a token control message to a node $\left(N_{j}\right)$. In case the request message is lost, i.e., the timer attached to this request message has expired and no reply message has received from the parent, then the child node resends ( maximum 3 times ) the request message to its parent nodes. In another scenario, if the token message is lost then the parent node resends ( maximum 3 times ) the token message to its child nodes. The detail control flow for receiving the token is shown in figure 3.

Figure 4 shows that node 9 receives a token from its parent node 5 which it uses to transmit data packets towards the sink 


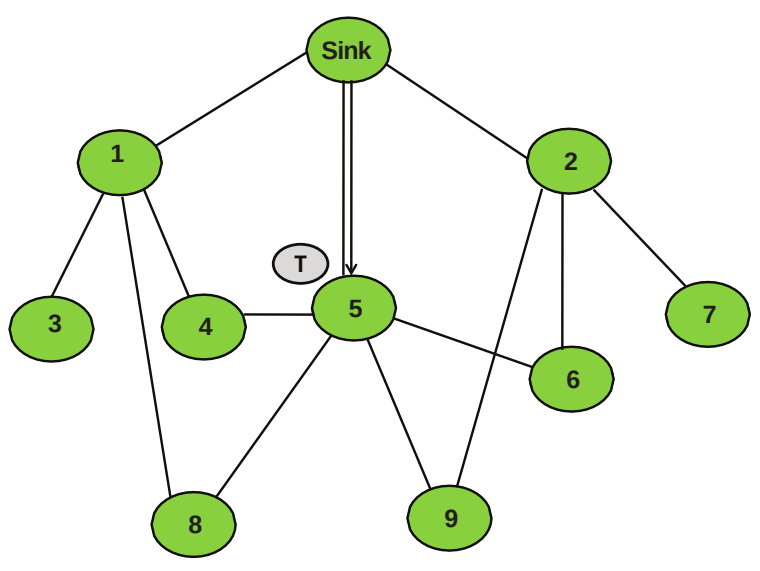

Fig. 5. Intermediate parent node receives token message from sink node

node. The node can transmit data till the time it holds the token. This token is given to the child node by its parent node only for a limited amount of time as described in Algorithm 2.

After receiving a token control message from parent nodes $P_{j 1}, P_{j 2}$ or both, the child node $\left(N_{j}\right)$ sends a reply control message to its parents. This reply control message is of two types, viz. acceptance reply message and rejection reply message. The acceptance reply message is sent to the parent node whose token arrives first at the child node to initiate data transmission. Whereas, the rejection reply message is sent to the parent node whose token arrived later at the child node as it has already accepted one token before from another parent node. The token rejected by a child node can be reused at the parent node for any other request generated by another child node in the same network. If any of the reply messages sent from the child node is lost, i.e., the timer attached to the reply message has expired and no acknowledgment message from the parent has been received, then the child node needs to resend (maximum 3 times) the same to its own parents. The above description for reply packet management is described in Algorithm 3. On receiving the acceptance reply message or rejection reply message from a child node $\left(N_{j}\right)$, both of its parent nodes $\left(P_{j 1}\right.$ and $\left.P_{j 2}\right)$ send an acknowledgment message to the child node $\left(N_{j}\right)$. On receiving the acknowledgment message from the parent node whose token is accepted $\left(P_{j 1}\right)$, the child node $\left(N_{j}\right)$ starts transmitting data packets to the same parent node $\left(P_{j 1}\right)$. If the acknowledgment message sent from the parent whose token is accepted by the child node is lost, then the data transmission cannot be possible between the child node and parent node, and the parent node needs to retransmit the acknowledgment message in order to start data transmission. The complete acknowledgment management is described in Algorithm 4. The token received by the child node has to be returned back with the last data packet to its parent node.

Figure 5 shows that the parent node also waits for a fixed time (Timer 4) or till the data queue (buffer) is full. Then the data packets get accumulated at each intermediate node which sends a request to its own parents for transmitting the accumulated data packets received from its child node.

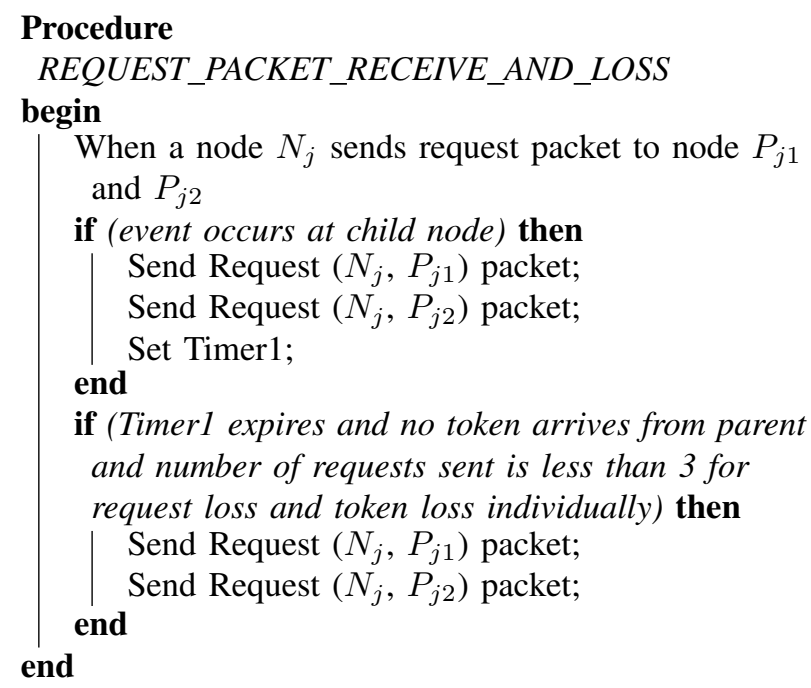

Algorithm 1: Request Packet Management Algorithm

In order to reduce end-to-end latency in data delivery, we need to handle the loss of control messages which is done in the proposed protocol as follows:

1) When either the request message or the token control message sent by child node is lost, the child node waits for a certain amount of time, i.e., till timer expires, and then resends the request packet to the parent nodes. A request to the parent nodes can be sent for maximum three times for the same data packet.

2) When the child node receives the token sent by both the parents, it sends an acceptance reply message to the parent nodes whose token message is accepted and rejection reply message to the parent node whose token message has been rejected. After receiving the reply messages by both the parent nodes, they send acknowledgment messages to the child nodes. On receiving the acknowledgment messages, the child node starts transmitting the data packets to the parent node whose token has been accepted.

3) In case the reply message sent by the child node gets lost, the parent nodes re-generate a token after the timer expires and sends it again to the child node. The child node on receiving new tokens, checks if it already has a token or not. If it already has a token from any of the parent nodes, it sends a rejection reply message to the concerned parent node. This re-sending of reply message can be done for a maximum three times in case of loss of reply message.

4) If the reply messages sent by the child node are received by the parent nodes but the acknowledgment packet sent by the parent nodes get lost, the child node waits for the timer to expire and then sends the reply message again to the parent node whose token has been accepted. This reply message can also be sent for maximum three times after certain time intervals in case of loss of acknowledgment messages. 

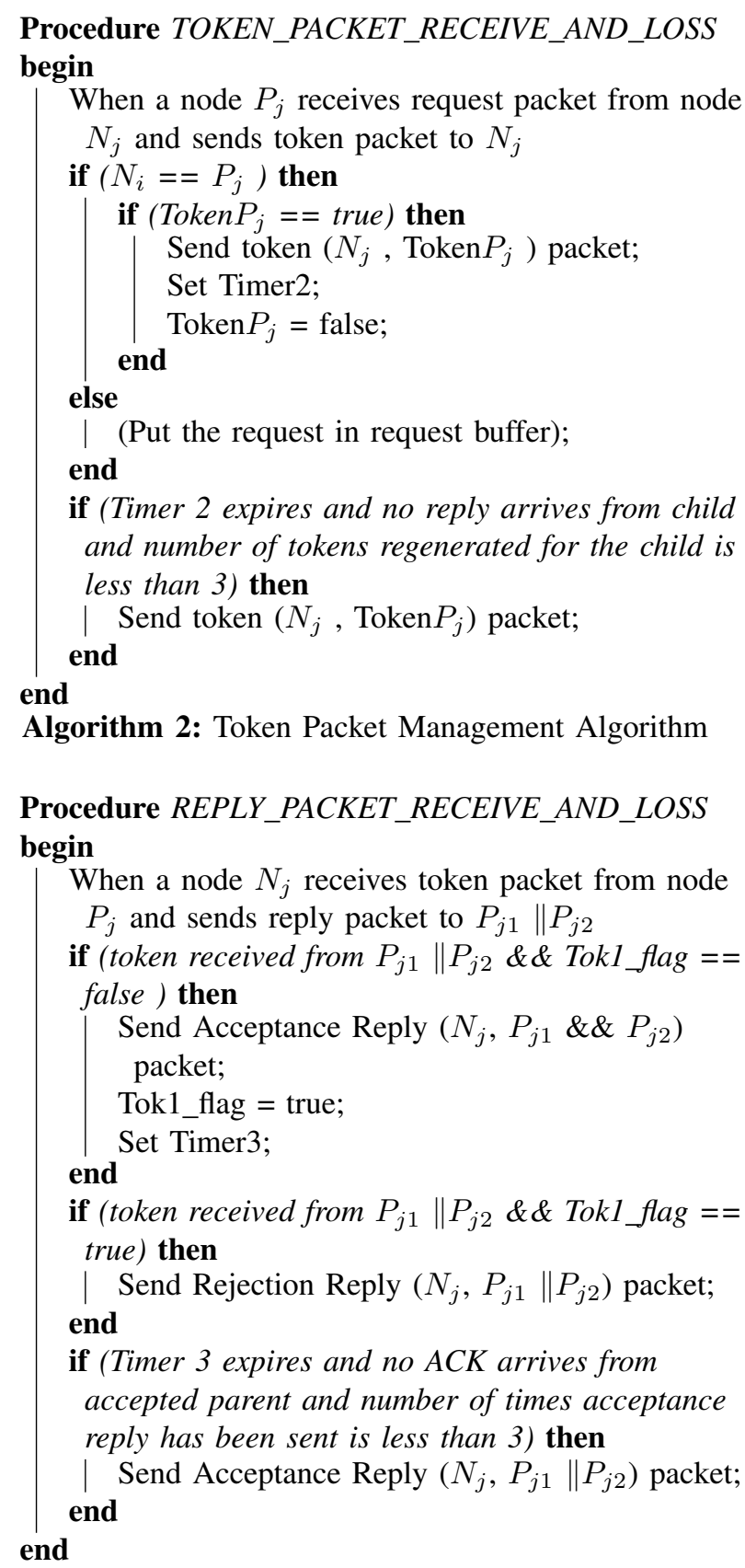

Algorithm 3: Reply Packet Management Algorithm

\section{Data Transmission Phase}

When node $N_{j}$ wants to send data to the sink node, it first acquires token from its immediate parent $P_{j}$ and keeps it for a limited period of time for data transmission and after that child node needs to return back the token. When the node $N_{j}$ needs to send or forwards a data packet, the following steps are executed:

1) As soon as the child node $N_{j}$ receives the TOKEN from one of its parents, it sends reply message to both of its parent nodes informing that $N_{j}$ acquired the Token of $P_{j 1}$. On receiving ACK message from the parent node $P_{j 1}$, it starts sending data packets from its buffer to parent $P_{j 1}$. The parent keeps the token field information as false until the timer expires or node $N_{j}$ has no data

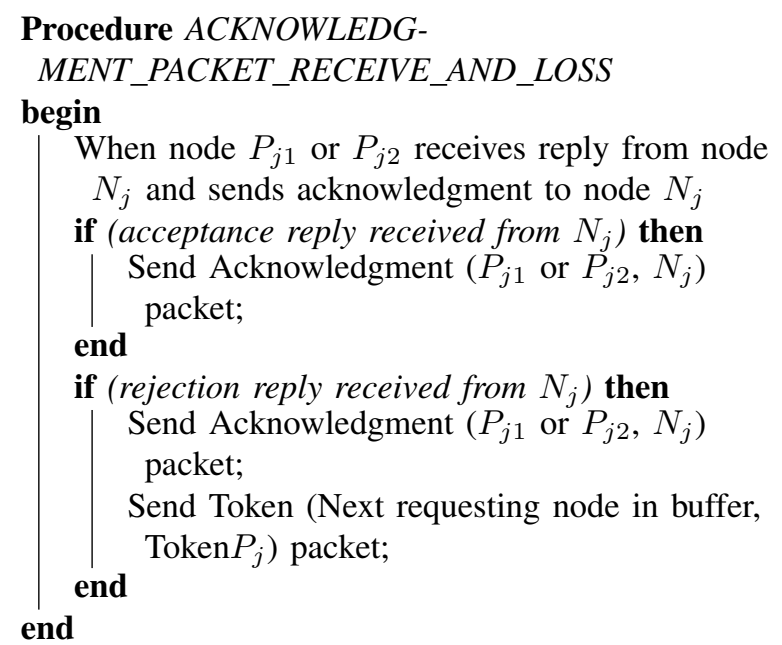

Algorithm 4: Acknowledgment Packet Management Algorithm

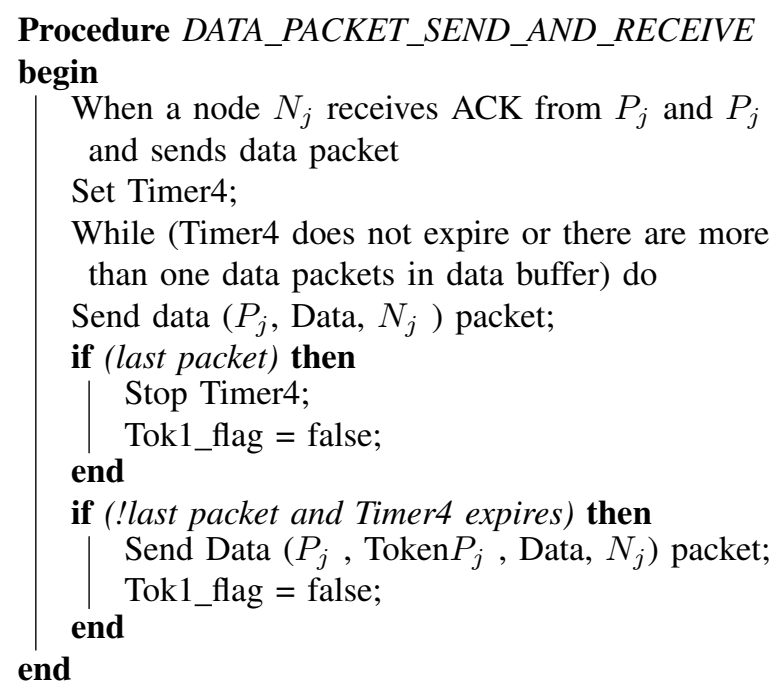

Algorithm 5: Data Packet Transmission Algorithm

to transmit, whichever occurs first.

2) On receiving the data packets, parent $P_{j 1}$ puts these packets in its data buffer. Once the data buffer gets full, the accumulated data packets are transmitted through the intermediate nodes in the network. The parent node gets back its token along with the last data packet it receives. It may provide the same token to some other child node if requested. If node $P_{j 1}$ is not a sink node, then it sends a request packet to its parent nodes in the same network in order to forward the data packets stored in its data buffer.

The above steps are repeated until the data is received by the sink node. The complete description related to data transmission is given in Algorithm 5.

\section{Simulation Results}

An extensive simulation study of our proposed protocol is carried out using Castalia simulator 3.2 [45] to evaluate its performance and also compare its performance with that of Multi-token based MAC protocol [11]. In this simulation 


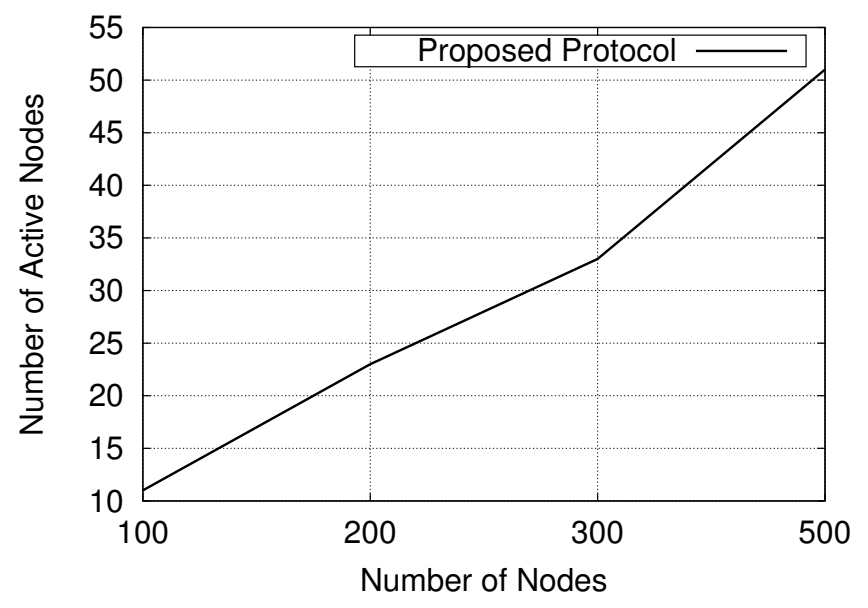

Fig. 6. Number of active nodes in different size WSNs in the proposed protocol

study, the number of sensor node with a range from 100 to 500 is deployed in a uniform random manner. In addition to this, specific parameters like transmission rate, transmission range, transmission power, sensitivity are taken into account as per the values are given in the CC2420 datasheet [46] and TelosB datasheet [47]. For our simulation, we use the following parameters as given in table I.

TABLE I

PARAMETERS AND THEIR CORRESPONDING VALUES USED FOR SIMULATION

\begin{tabular}{ll}
\cline { 2 - 2 } Parameter & Value \\
\cline { 2 - 2 } Number of Nodes & $100-500$ \\
Frequency & $2.4 \mathrm{GHz}$ \\
Deployment & Uniform \\
Bandwidth & $250 \mathrm{kbps}$ \\
Modulator Type & PSK \\
Transmission Power & $-3 \mathrm{dBm}$ \\
Packet Rate & $0.1 / \mathrm{Sec}$. \\
Node Density & $36 \mathrm{Sqmtr}$. \\
\hline
\end{tabular}

In this simulation, we have not configured any existing MAC and routing protocol as we are implementing both MAC and routing protocol of our own. The deployment of the sensor node are randomly uniform in nature. Each node communicates with the sink node using multi-hop network topology. The proposed protocol is executed periodically, and during each turn, the nodes in WSN can sense the environment and send the sensed data to the sink node using the hierarchical structure.

Figure 6 represents the number of active nodes present in variable size WSNs using our proposed distributed approach. This figure depicts that as the size of the WSN increases, the number of active nodes increases. This indicates that the distributed approach maintains the active nodes in different size of WSNs.

Figure 7 shows comparative study on the number of active nodes identified in the proposed protocol and existing multi-

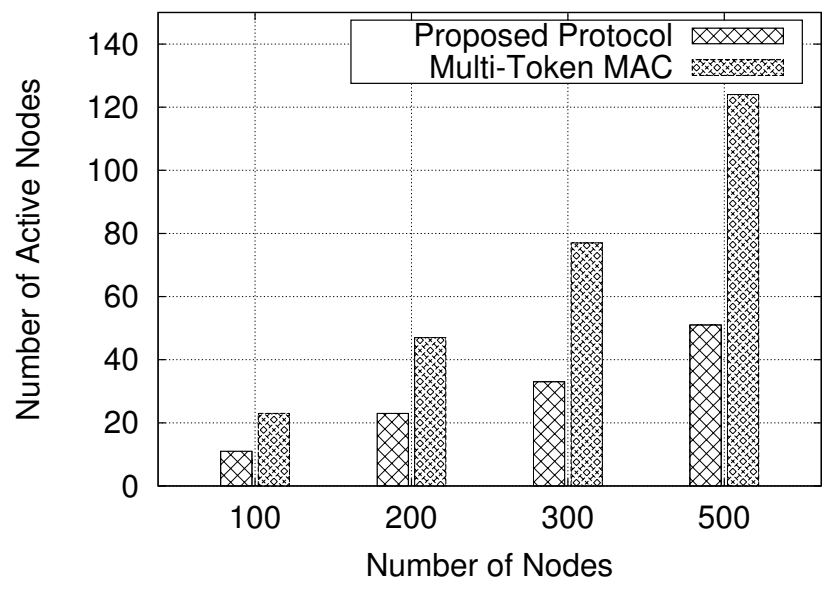

Fig. 7. Number of active nodes in the proposed protocol vs Multi-token MAC protocol

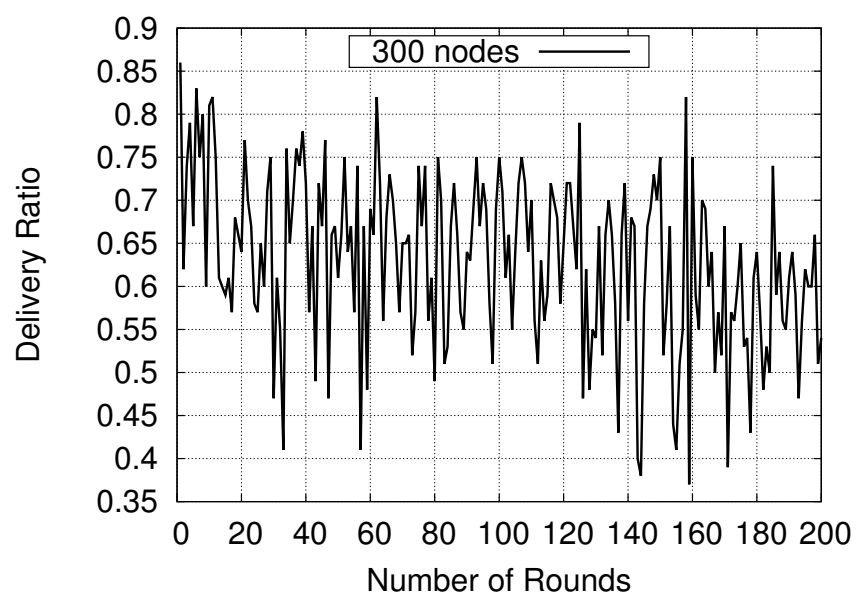

Fig. 8. Delivery ratio for 300 nodes in 200 rounds in proposed protocol

token based MAC protocol [11] for different size WSNs. It is observed that when the size of the network gets increased, the number of active nodes in our proposed protocol always remain less than that of the existing multi-token based MAC protocol for different size of WSNs.

Figure 8 shows the data packet delivery ratio during data transmission between the source and the sink node for the proposed protocol using 300 nodes in the network with a number of rounds varying from 0 to 200 . The data delivery ratio varies in different rounds due to varying congestion in the WSN. The delivery ratio also varies due to the change in the number of active nodes in different rounds as the network structure is reconstructed in each round. This results in efficient energy consumption over the whole WSN during data transmission and it also creates a congestion free data transmission through the routing path.

Figure 9 shows the comparative study on end-to-end data delivery ratio of the proposed protocol, Multi-token based MAC protocol, and ETR protocol. It can be depicted from the figure that the delivery ratio of these protocols decreases with respect to an increase in node density. In case of multi- 


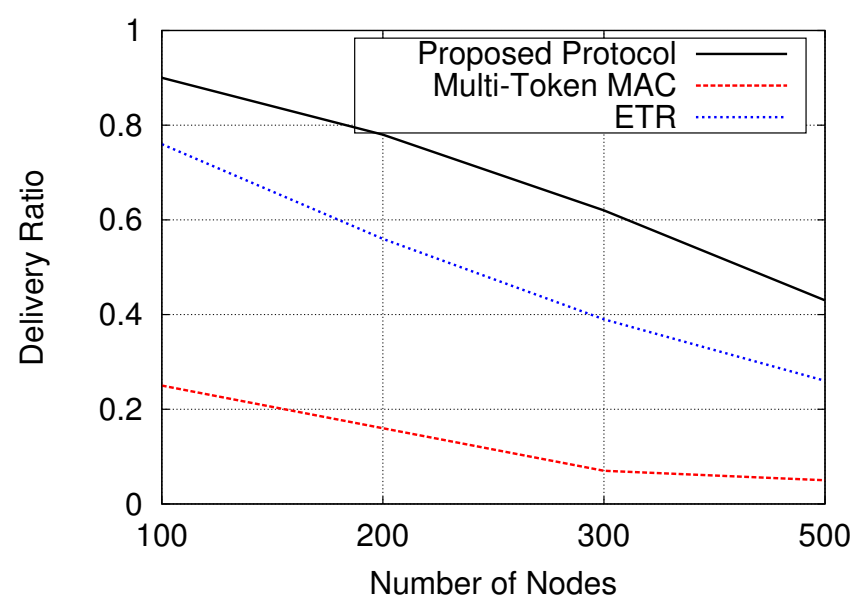

Fig. 9. End-to-end data delivery ratio in proposed protocol vs Multi-token MAC protocol vs ETR Protocol

token based MAC protocol, with an increase in the number of nodes more number of intermediate nodes, are available only for forwarding the data packet towards the sink node. This, in turn, increases the congestion in the network and consequently increases data loss. As only request messages and token control messages are used in multi-token based MAC protocol, it can manage to have good data delivery ratio at the cost of high energy consumption over a varying number of nodes. At the same time, in case of ETR protocol, an alternate shortest path is found out in addition to the parent \& child link in order to forward the data packet to the sink node. So, the alternate path used in ETR protocol helps to reduce the network load and network traffic over parent and child link, however, the protocol consumes more energy to find out the alternate path. Nevertheless, in case of the proposed protocol, the new control messages like reply and acknowledgment messages along with the existing request and token control messages improve the data delivery ratio of the proposed protocol which is higher in comparison to multitoken based MAC protocol and ETR protocol in different sized WSNs.

Figure 10 shows the hop-to-hop data delivery ratio of AS2 MAC protocol and the proposed protocol. It can be easily understood from the figure that the data delivery ratio of AS2 MAC protocol decreases with an increase in node density over a varying number of nodes. In AS2 MAC protocol, as both packet transmission and receive work separately to avoid interference of data packets in WSN, hence, with the increase in node density, the MAC protocol fails to get nonoverlapping time spans leading to low data delivery ratio. However, in the case of the proposed protocol, with the help of new control messages like reply and acknowledgment, the data delivery ratio is comparatively better in respect to each hop transmission in the entire network. It is also evident from the figure that the data delivery ratio is consistent with respect to increasing node density over the varying size of the network as compared to AS2 MAC protocol.

As far as data packet delivery ratio in WSNs is concerned, the energy consumption of each node during transmission is

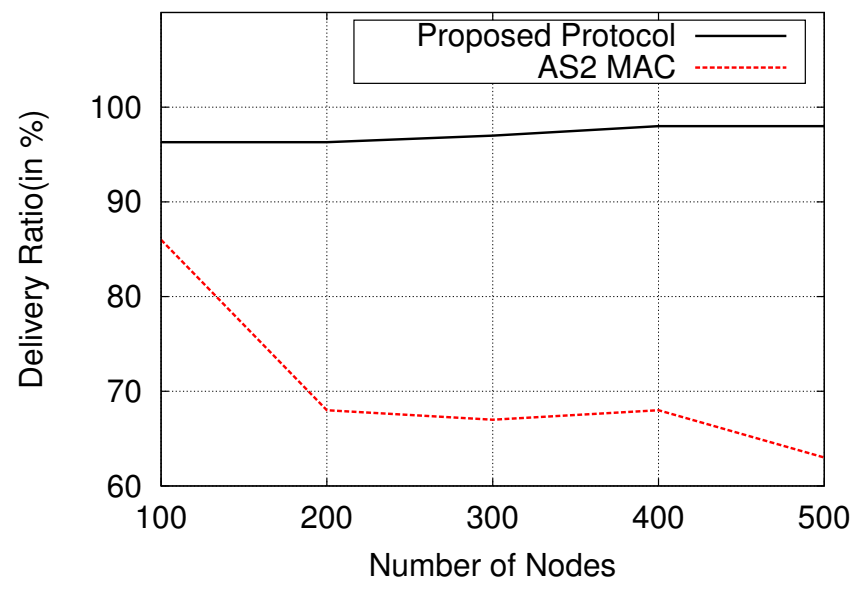

Fig. 10. Hop-to-hop data packet delivery ratio in proposed protocol vs AS2 MAC protocol

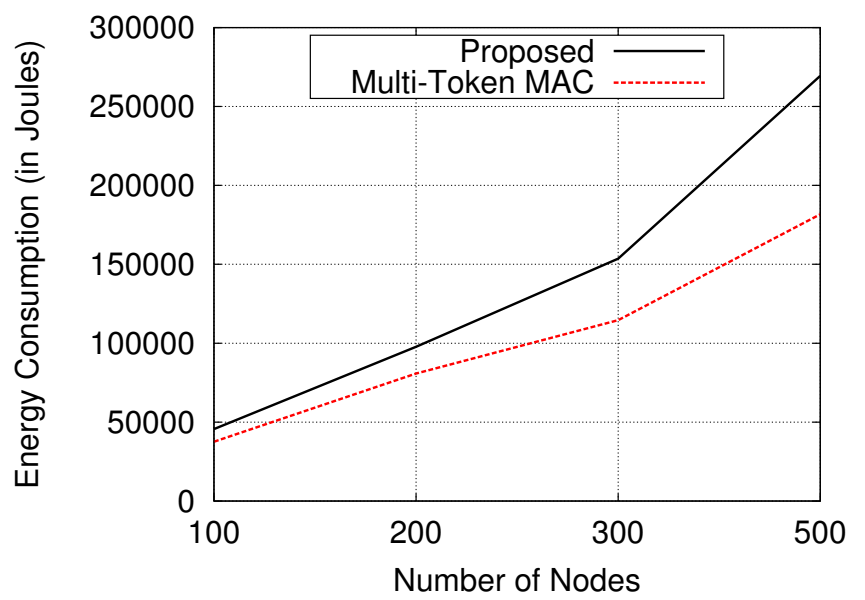

Fig. 11. Total energy consumption in proposed protocol vs Multi-token MAC protocol

one of the important requirement to prolong the network connectivity in the network. Figure 11 describes the understanding about the effective energy consumption of proposed protocol and Multi-token based MAC protocol in different sized WSNs. It can be noted that the proposed protocol consumes more energy as compared to the Multi-token based MAC protocol. This is because of the high data delivery ratio of the proposed protocol as compared to Multi-token based MAC protocol as depicted in Figure 9. As the proposed protocol comparatively exchanges more number of control messages for collision free data transmission, the energy consumption of each node consequently increases leading to an increase in total energy consumption of the WSNs. However, the rate of increase in the delivery ratio is quite high as compared to the rate of increase in energy consumption.

Figure 12 represents the number of sleep nodes, active nodes, and dead nodes in a 500 node size WSN. Here, the nodes which do not have the energy to run further are referred to as dead nodes. From this figure, it is clearly observed that the nodes start dying at around $165^{\text {th }}$ round. The nodes 


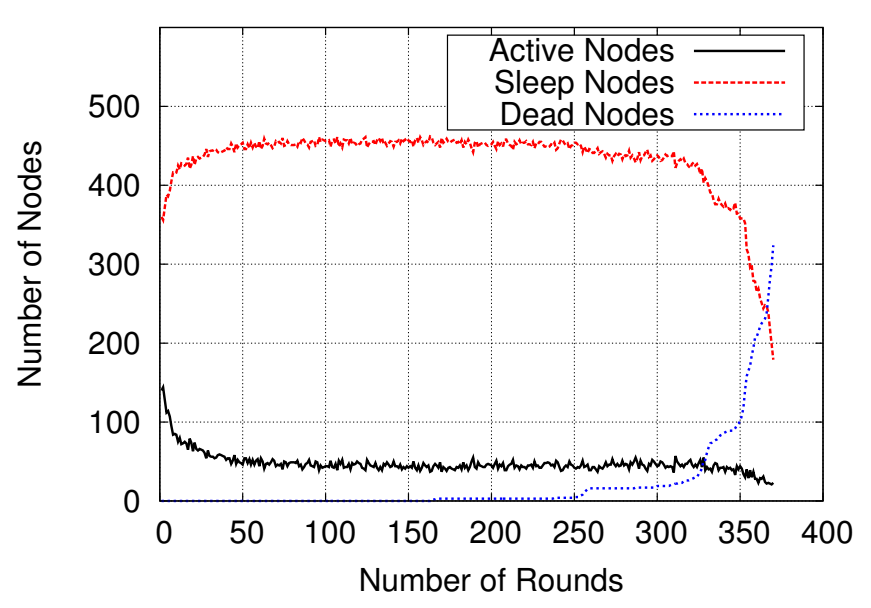

Fig. 12. Number of active nodes, dead nodes, and sleep nodes in proposed approach

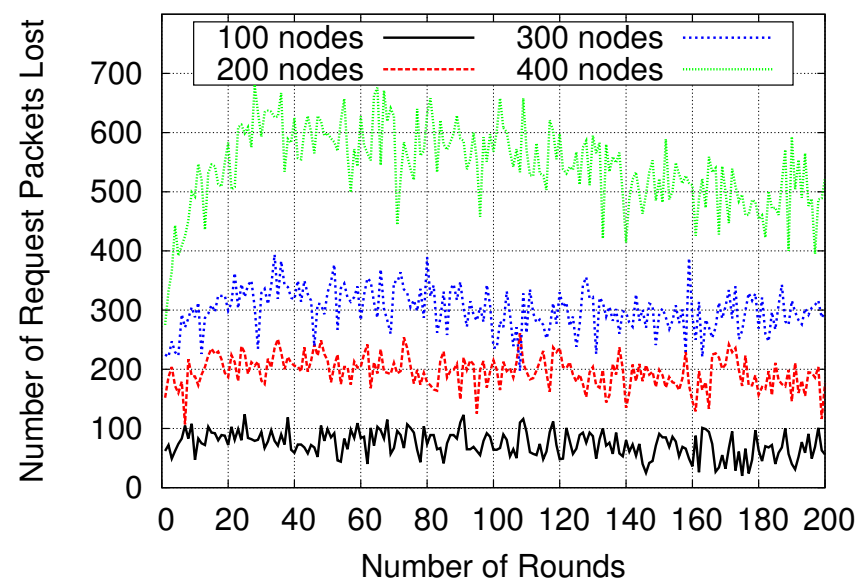

Fig. 13. Number of request packets lost in different size WSNs

prominently start dying at around $253^{\text {rd }}$ round. Also, the number of active and sleep nodes are nearly fixed till $253^{\text {rd }}$ round. But, after $253^{\text {rd }}$ round, the number of sleep nodes that suddenly starts decreasing which in turn have high energy consumption in the network. The number of active nodes is also decreasing after $319^{\text {th }}$ round which in turn affects the connectivity in the network.

In order to initiate data transmission, request packets are sent to the parent nodes. In the proposed protocol, if any of the request packets is lost, it can be resent (maximum 3 times) to the parent node. However, resending of request packet affects the data transmission in two different manners, i.e., (i) increase in latency and (ii) the high energy consumption which in turn decreases network lifetime. Figure 13 shows variation in loss of request packets in different rounds with respect to the different size of WSNs. From the figure, it can be clearly observed that the number of lost request packets increases linearly with an increase in the number of nodes in the network. This is because, as the size of WSN increases, congestion in the network increases which in turn increases the loss in request packets.

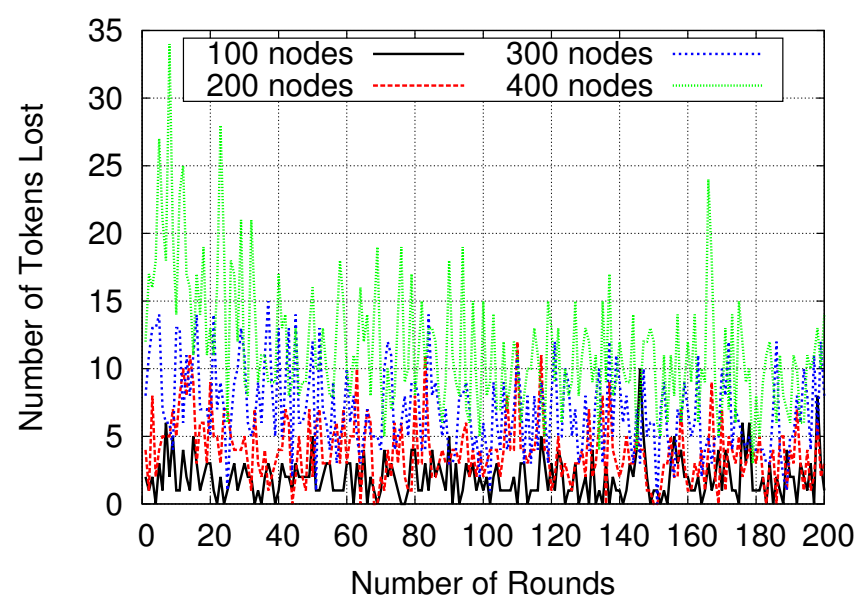

Fig. 14. Number of tokens lost in different size WSNs

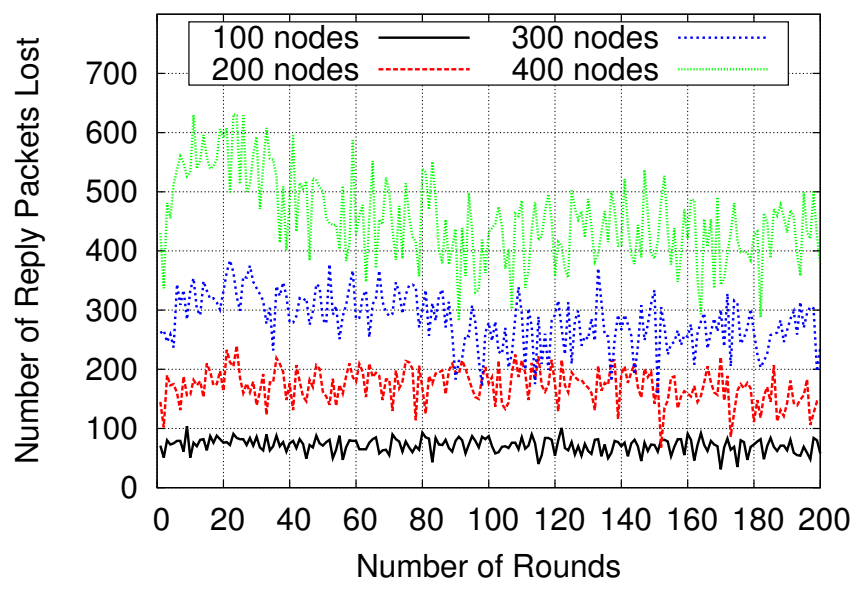

Fig. 15. Number of reply packets lost in different size WSNs

In order to transmit data by a node to its immediate parent, each node must have the token control message from its corresponding parents. If a token gets lost before reaching to the child node, then as per the proposed approach, the token must be resent (maximum 3 times) which increases the energy consumption in the network. Figure 14 shows the number of tokens lost in different rounds with respect to the different size of WSNs. This figure clearly shows that with the increase in node density, the loss of token control message is also increased.

A reply control message should be sent to both the parents from a child node to confirm that the token is accepted by the child node in order to start data transmission. In the case the reply packet is lost, it can be present for maximum 3 times according to the proposed approach but resending the packet always requires high energy consumption. Figure 15 gives the understanding of reply packet lost in each round with varying node density. It is observed that with the increase in the number of nodes, the number of reply packet loss is also increased.

An acknowledgment control message is always there from parents to child nodes in order to confirm data transmission. If 


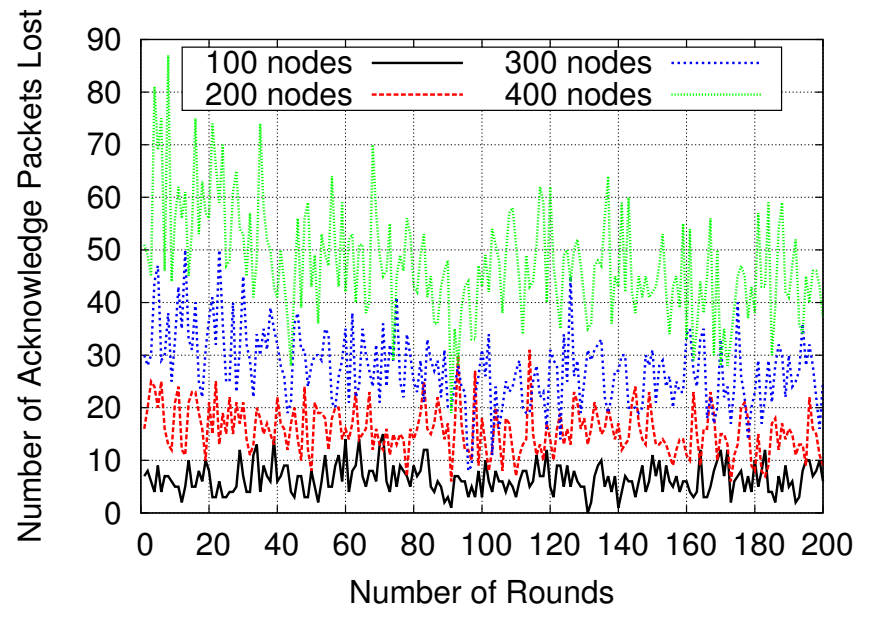

Fig. 16. Number of ACK packets lost in different size WSNs

an acknowledgment packet gets lost, it can be resent up to 3 times at the cost of high energy consumption. Figure 16 shows the number of acknowledgment packet loss in different rounds with respect to the varying number of nodes in the WSN. It is clearly observed that the number of acknowledgment packet loss increases as the node density increases in the network.

\section{CONCLUSiOnS AND Future Work}

In this paper, we proposed a MAC-cum-routing protocol that provides collision free data transmission to achieve maximum throughput with respect to packet delivery. To fulfill the above requirement, the Proposed approach used a message passing technique to disseminate multiple tokens among the active sensor nodes in a distributed manner, maintain connectivity among all the active nodes, and achieve collision free data transmission even when a node in the network runs out of energy. The proposed protocol is flexible enough to cope up with node failure or network hole by maintaining multiple parents. At the same time, it also maintains the energy consumption evenly over the whole WSN. The distributed approach for the creation of network structure to implement the proposed MACcum-routing protocol gives better throughput using a robust multi-token management scheme. However, the performance of the distributed approach can be further enhanced by the use of multiple sink nodes, which can be considered as future work.

\section{REFERENCES}

[1] J. Yick, B. Mukherjee, and D. Ghosal, "Wireless sensor network survey," Comput. Netw., vol. 52, no. 12, pp. 2292-2330, Aug. 2008, DOI: 10.1016/j.comnet.2008.04.002.

[2] I. F. Akyildiz, W. Su, Y. Sankarasubramaniam, and E. Cayirci, "Wireless sensor networks: A survey," Comput. Netw., vol. 38, no. 4, pp. 393-422, Mar. 2002, DOI: 10.1016/S1389-1286(01)00302-4.

[3] W. Ye, J. Heidemann, and D. Estrin, "An energy-efficient mac protocol for wireless sensor networks," in Proceedings.Twenty-First Annual Joint Conference of the IEEE Computer and Communications Societies, vol. 3, June 2002, pp. 1567-1576, DOI: 10.1109/INFCOM.2002.1019408.

[4] R. Munadi, A. E. Sulistyorini, F. U. F. S, and T. Adiprabowo, "Simulation and analysis of energy consumption for s-mac and t-mac protocols on wireless sensor network," in 2015 IEEE Asia Pacific Conference on Wireless and Mobile (APWiMob), Aug 2015, pp. 142-146, DOI: 10.1109/APWiMob.2015.7374976.
[5] C. Portillo, J. Martinez-Bauset, and V. Pla, "Modelling of s-mac for heterogeneous wsn," in 20189 th IFIP International Conference on New Technologies, Mobility and Security (NTMS), Feb 2018, pp. 1-6, DOI: 10.1109/NTMS.2018.8328705.

[6] S. Du, A. K. Saha, and D. B. Johnson, "Rmac: A routing-enhanced dutycycle mac protocol for wireless sensor networks," in IEEE INFOCOM 2007 - 26th IEEE International Conference on Computer Communications, May 2007, pp. 1478-1486, DOI: 10.1109/INFCOM.2007.174.

[7] K. T. Cho and S. Bahk, "He-mac: Hop extended mac protocol for wireless sensor networks." in GLOBECOM. IEEE, 2009, pp. 1-6, DOI: 10.1016/j.comnet.2012.01.001.

[8] S. Ray, S. Dash, N. Tarasia, A. Ajay, and A. R. Swain, "Energy efficient token based mac protocol for wireless sensor networks," International Journal of Computer Science and Information Technologies(IJCSIT), vol. 2, no. 2, pp. 747-753, Dec. 2011.

[9] S. Ray, S. Dash, N. Tarasia, A. Ajay, and A. Swain, "Congestion-less energy aware token based mac protocol integrated with sleep scheduling for wireless sensor networks," Proceedings of the World Congress on Engineering, vol. II, pp. 755-1760, 072011.

[10] A. Ajay, N. Tarasia, S. Dash, R. Soumya, and A. R. Swain, "Fault tolerant multilevel routing protocol with sleep scheduling (fms) for wireless sensor networks," European Journal of Scientific Research(EJSR)., vol. 51, no. 1, pp. 97-111, june 2011.

[11] S. Dash, A. R. Swain, and A. Ajay, "Reliable energy aware multi-token based mac protocol for wsn." in AINA. IEEE, 2012, pp. 144-151, DOI: 10.1109/AINA.2012.61.

[12] S. Ray, S. Dash, N. Tarasia, A. Ajay, and A. R. Swain, "A self organising message passing approach for data accuracy in mac protocol for wsns," in International Conference on Wireless and Optical Communications (ICWOC). Zhengzhou, China: IEEE, May 2011, pp. 483-488.

[13] T. van Dam and K. Langendoen, "An adaptive energy-efficient mac protocol for wireless sensor networks," in Proceedings of the 1st International Conference on Embedded Networked Sensor Systems, ser. SenSys '03. New York, NY, USA: ACM, 2003, pp. 171-180, DOI: $10.1145 / 958491.958512$

[14] S. Thaskani, K. V. Kumar, and G. R. Murthy, "Energy efficient crosslayer design protocol by using token passing mechanism for wsn," pp. 572-575, March 2011, DOI: 10.1109/ISCI.2011.5958979.

[15] I. Dbibih, I. Iala, D. Aboutajdine, and O. Zytoune, "Ass-mac: Adaptive sleeping sensor mac protocol designed for wireless sensor networks," in 2016 International Conference on Information Technology for Organizations Development (IT4OD), March 2016, pp. 1-5, DOI: 10.1109/IT4OD.2016.7479301.

[16] M. I. Khalil, M. A. Hossain, M. J. Haque, and M. N. Hasan, "Eerc-mac: Energy efficient receiver centric mac protocol for wireless sensor network," in 2017 IEEE International Conference on Imaging, Vision Pattern Recognition (icIVPR), Feb 2017, pp. 1-5, DOI: 10.1109/ICIVPR.2017.7890861.

[17] L. Lin, N. B. Shroff, and R. Srikant, "Energy-aware routing in sensor networks: A large system approach," Ad Hoc Netw., vol. 5, no. 6, pp. 818-831, 2007, DOI: 10.1016/j.adhoc.2007.02.009.

[18] M. Z. n. Zamalloa, K. Seada, B. Krishnamachari, and A. Helmy, "Efficient geographic routing over lossy links in wireless sensor networks," ACM Trans. Sen. Netw., vol. 4, no. 3, pp. 12:1-12:33, Jun. 2008, DOI: $10.1145 / 1362542.1362543$

[19] R. Simón Carbajo, M. Huggard, and C. McGoldrick, "An end-to-end routing protocol for peer-to-peer communication in wireless sensor networks," in MiNEMA '08: Proceedings of the 6th workshop on Middleware for network eccentric and mobile applications. New York, NY, USA: ACM, 2008, pp. 5-9, DOI: 10.1145/1435467.1435469.

[20] J. N. Al-Karaki and A. E. Kamal, "Routing techniques in wireless sensor networks: A survey," Wireless Commun., vol. 11, no. 6, pp. 6-28, Dec. 2004.

[21] T. Liu, Y. Gu, and Z. Hu, "Energy equalizing routing algorithm for wireless sensor networks," in Computational Intelligence and Design, 2009. ISCID '09. Second International Symposium on, vol. 1, Dec. 2009, pp. 546-549, DOI: 10.1109/ISCID.2009.143.

[22] C. Luo, F. Wu, J. Sun, and C. W. Chen, "Compressive data gathering for large-scale wireless sensor networks," in Proceedings of the 15th Annual International Conference on Mobile Computing and Networking, ser. MobiCom '09, 2009, pp. 145-156.

[23] W. Qiu, E. Skafidas, and P. Hao, "Enhanced tree routing for wireless sensor networks," Ad Hoc Netw., vol. 7, no. 3, pp. 638-650, May 2009.

[24] S. Rani, J. Malhotra, and R. Talwar, "Energy efficient chain based cooperative routing protocol for wsn," Appl. Soft Comput., vol. 35, no. C, pp. 386-397, Oct. 2015 . 
[25] F. Ye, A. Chen, S. Lu, and L. Z. 0001, "A scalable solution to minimum cost forwarding in large sensor networks." in ICCCN. IEEE, 2001, pp. 304-309, DOI: 10.1109/ICCCN.2001.956276.

[26] A. A. Masri, G. Y. Delisle, and N. Hakem, "Hierarchical routing protocol for multi-level heterogeneous sensor network," in 2016 16th Mediterranean Microwave Symposium (MMS), Nov 2016, pp. 1-4, DOI: 10.1109/MMS.2016.7803853.

[27] R. Singh, B. K. Rai, and S. K. Bose, "A contention based routing enhanced mac protocol for transmission delay reduction in a multi-hop wsn," in TENCON 2017 - 2017 IEEE Region 10 Conference, Nov 2017, pp. 398-402, DOI: 10.1109/TENCON.2017.8227897.

[28] M. Razzaq, G. Kwon, and S. Shin, "Energy efficient dijkstra-based weighted sum minimization routing protocol for wsn," in 2018 Third International Conference on Fog and Mobile Edge Computing (FMEC), April 2018, pp. 246-251.

[29] L. Farhan, O. Kaiwartya, L. Alzubaidi, W. Gheth, E. Dimla, and R. Kharel, "Toward interference aware iot framework: Energy and geolocation-based-modeling," IEEE Access, vol. 7, pp. 56 617-56 630, 2019.

[30] R. Subramanian and F. Fekri, "Sleep scheduling and lifetime maximization in sensor networks: fundamental limits and optimal solutions," in IPSN '06: Proceedings of the 5th international conference on Information processing in sensor networks, 2006, pp. 218-225, DOI: 10.1145/1127777.1127813.

[31] E. Bulut and I. Korpeoglu, "Sleep scheduling with expected common coverage in wireless sensor networks," Wirel. Netw., vol. 17, no. 1, pp. 19-40, Jan. 2011.

[32] D. Shuman and M. Liu, "Optimal sleep scheduling for a wireless sensor network node," in Signals, Systems and Computers, 2006. ACSSC '06. Fortieth Asilomar Conference on, 2006, pp. 1337-1341, DOI: 10.1109/ACSSC.2006.354974.

[33] T. Kumberg, M. Schink, L. Reindl, and C. Schindelhauer, "Trome: A simple and energy efficient tree routing protocol for low-power wake-up receivers," Ad Hoc Networks, 02 2017, DOI 10.1016/j.adhoc.2017.02.003.

[34] R. Shah and J. Rabaey, "Energy aware routing for low energy ad hoc sensor networks," in Wireless Communications and Networking Conference, 2002. WCNC2002. 2002 IEEE, vol. 1, Mar 2002, pp. 350355 vol.1, DOI: 10.1109/WCNC.2002.993520.

[35] M.-L. Pham, D. Kim, S. Yoo, and Y. Doh, "Power aware chain routing protocol for data gathering in sensor networks." IJDSN, no. 2, pp. 253 267, DOI: 10.1080/15501320590966503.

[36] L. Hong and J. Yang, "An energy-balance multipath routing based on rumor routing for wireless sensor networks," in 2009 Fifth International Conference on Natural Computation. IEEE Computer Society, 2009, pp. 87-91, DOI: 10.1109/ICNC.2009.276.

[37] N. Chakchouk, B. Hamdaoui, and M. Frikha, "Wcds-dcr: an energyefficient data-centric routing scheme for wireless sensor networks." Wireless Communications and Mobile Computing, no. 2, pp. 195-205, DOI: $10.1002 / \mathrm{wcm} .956$.

[38] R. E. Ahmed, "A fault-tolerant, energy-efficient routing protocol for wireless sensor networks," in 2015 International Conference on Information and Communication Technology Research (ICTRC), May 2015, pp. 175-178, DOI: 10.1109/ICTRC.2015.7156450.

[39] N. F. Shah, Amritanjali, S. Gautam, and D. Gosain, "Eera: Energy efficient reliable routing algorithm for wsn," pp. 1-5, Aug 2016, DOI: 10.1109/IICIP.2016.7975384

[40] T. W. Tukisi, T. N. D. Mathaba, and M. O. Odhiambo, "Multi-hop pso based routing protocol for wireless sensor networks with energy harvesting," in 2019 Conference on Information Communications Technology and Society (ICTAS), March 2019, pp. 1-6.

[41] S. Dash, S. S. Mallick, R. C. Hansdah, and A. R. Swain, "A distributed approach to construct hierarchical structure for routing with balanced energy consumption in wsns," in 2015 IEEE 29th International Conference on Advanced Information Networking and Applications, March 2015, pp. 382-388, DOI: 10.1109/AINA.2015.210.

[42] A. R. Swain, R. C. Hansdah, and V. K. Chouhan, "An energy aware routing protocol with sleep scheduling for wireless sensor networks," in Proceedings of the 2010 24th IEEE International Conference on Advanced Information Networking and Applications, ser. AINA '10, 2010, pp. 933-940.

[43] L. Anchora, A. Capone, V. Mighali, L. Patrono, and F. Simone, "A novel mac scheduler to minimize the energy consumption in a wireless sensor network," Ad Hoc Netw., vol. 16, pp. 88-104, May 2014, DOI: 10.1016/j.adhoc.2013.12.002.

[44] W. Qiu, E. Skafidas, and P. Hao, "Enhanced tree routing for wireless sensor networks," Ad Hoc Netw., vol. 7, no. 3, pp. 638-650, May 2009, DOI: 10.1016/j.adhoc.2008.07.006.
[45] "Castalia a simulator for wireless sensor networks," http://castalia.npc.nicta.com.au/pdfs/Castalia User Manual.pdf.

[46] "Cc2420 data sheet," http://www.stanford.edu/class/cs244e/ papers/cc2420.pdf.

[47] "Telosb data sheet," http://www.xbow.com/Products/ Product_pdf_files/Wireless_pdf/TelosB_Datasheet.pdf.

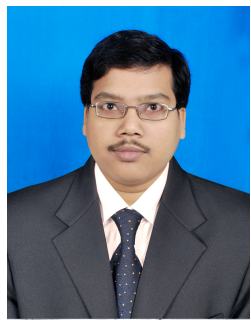

Subhasis Dash received his M.Tech degree in Computer Science and Engineering from KIIT University, Bhubaneswar, India in 2006. Currently, he is working as an assistant professor in the School of Computer Engineering, KIIT Deemed To Be University, Bhubaneswar, India. His research interests include wireless sensor networks, distributed computing, and operating systems.

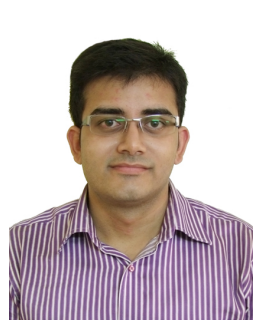

Kumar Saras received his B. Tech. degree in Information Technology from School of Computer Engineering, Kalinga Institute of Industrial Technology (KIIT) University, Bhubaneswar, Odisha, India His research interests are Wireless Sensor Networks, Internet of Things, Big Data, etc.

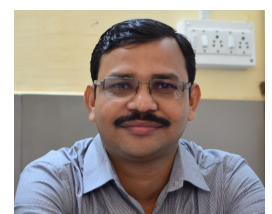

Manas Ranjan Lenka received his M.Tech degree in Computer Science and Engineering from BPUT University, Bhubaneswar, India in 2006. Currently, he is working as an assistant professor in the School of Computer Engineering, KIIT Deemed To Be University, Bhubaneswar, India. His research interests include wireless sensor networks, Data Analytics.

Amulya Ratna Swain received his M.E. Degree in Software Engineering from Jadavpur University, Calcutta, India in 2006. He received the Ph.D. degree in Computer Science from Indian Institute of Science, Bangalore, India, in 2013. Currently, he is working as an associate professor in the School of Computer Engineering, KIIT Deemed To Be University, Bhubaneswar, India. His research interests include wireless sensor networks, distributed computing, and operating systems. 